A. J. Sommese and A. Van de Ven

Nagoya Math. J.

Vol. 102 (1986), 79-90

\title{
HOMOTOPY GROUPS OF PULLBACKS OF VARIETIES
}

\author{
ANDREW JOHN SOMMESE AND A. VAN DE VEN
}

In $[2, \S 9]$ there is a general result of Fulton and Lazarsfeld relating the homotopy groups of a subvariety of $\boldsymbol{P}_{c}^{n}$ in a certain range of dimensions with those of its pullback under a holomorphic map in the corresponding range of dimensions. It is asked in $[2, \S 10]$ whether here is a corresponding result with $\boldsymbol{P}_{C}^{n}$ replaced by a general rational homogeneous manifold, $Y$, and with the range of dimensions alluded to above shifted by the ampleness of the holomorphic tangent bundle of $Y$ in the sense of [4]. In this paper we use the techniques of $[4,5,6,7]$ to answer this question in the affirmative.

Let us first recall the notion of $k$-ampleness for holomorphic vector bundles [4; see 1 also]. When $k=0$ this notion coincides with ampleness in the sense of Grothendieck-Hartshorne. Since all the bundles for which we need this notion are spanned, the definition takes a very simple form. Let $E$ be a holomorphic vector bundle on a compact complex manifold that is spanned at all points by global holomorphic sections. $E$ is $k$-ample if for each subvariety $Z \subseteq X$ such that $\left.E\right|_{Z}$ has a trivial quotient bundle, it is true that $\operatorname{dim} Z \leqslant k$.

(2.2) TheOREm. Let $f: W \rightarrow Y$ be a holomorphic map from a connected compact complex manifold $W$ to a connected rational homogeneous projective manifold $Y$. Assume that $f^{*} T_{Y}$, the pullback of the holomorphic tangent bundle of $Y$, is $k$ ample. Let $Z$ be a connected complex submanifold of $Y$. Let $d=\operatorname{dim} W-\operatorname{cod} Z-k$. If $d>0$ then $f^{-1}(Z)$ is connected and for all $a \in f^{-1}(Z)$

$$
f_{*}: \pi_{j}\left(W, f^{-1}(Z), a\right) \longrightarrow \pi_{j}(Y, Z, f(a))
$$

is an isomorphism if $j \leqslant d$, and a surjection if $j=d+1$.

A few remarks are in order.

Received September 3, 1984. 
In the case when $d=0$, the proof of the above theorem shows that $f^{-1}(Z)$ is non-empty.

The number $k$ that occurs in the above theorem is very computable. Let $t$ denote the ampleness of $T_{Y}$ and let $m$ denote the maximum of the fibre dimensions of the map $f$. Then $k \leqslant t+m$. For the Grassmannian, $\operatorname{Gr}(n, r)$, of the quotient $C^{r}$ 's of $C^{n}, t=r(n-r)-n+1$ and for the any smooth quadric $t=1$ (see [5,7]). For the general formula see [3].

Since the ampleness of $f^{*} T_{Y}$ takes more of the geometry of the map $f$ into account, it is often more useful than simply using the bound $t+m$. For example let $E$ be a $k$ ample bundle on a compact connected complex manifold $W$ that is spanned at all points by a vector space $V$ of global sections. Let $\operatorname{dim} V=n$ and let $f: W \rightarrow \operatorname{Gr}(n, \operatorname{rk} E)$ be the map associated to the evaluation map

$$
W \times V \longrightarrow E \longrightarrow 0 .
$$

Then $f^{*} T_{Y} \approx E \otimes F^{*}$ where $F$ is the kernel of the evaluation map (\#). From this we can conclude that $f^{*} T_{Y}$ is $k$ ample; this is usually much better than the $k$ estimated by $t+m$ above. For more details on this example and for an application to the Gauss mapping, see Section 3.

There is a whole literature on connectedness results (see [2]). In particular for general $Y$ as above, Faltings [1] has a connectedness result that allows $W$ to be singular; there is a discussion of this in [3].

Let us go over the contents of this paper in detail.

In Section 1 we consider a very general setup. We have a connected Lie group $G$ acting on a not necessarily compact complex manifold, $X$. We have two complex manifolds $B$ and $A$ on $X$. We assume that $B$ is compact and has a $k$ ample normal bundle. Except that $X$ is not necessarily homogeneous, this is the setup studied in $[6 ; \S 3]$. Let $\tilde{B}$ denote the family of intersections of $B$ with $G$ translates of $A$ :

$$
\tilde{B}=\{(g, a) \in G \times A \mid a g \in B\} .
$$

Using the results in [6] we show that the map $\tilde{B} \rightarrow G$ induced by the product projection $G \times A \rightarrow G$ has a long exact homotopy sequence like that of a fibre bundle in a certain range of dimensions. From this and elementary homotopy theory we get Theorem (1.1) which asserts that the map:

$$
\pi_{j}(A, A \cap B, a) \longrightarrow \pi_{j}\left(G \times A, \tilde{B}, a^{\prime}\right)
$$


induced by the inclusion $A \rightarrow\left(\operatorname{id}_{G}, A\right)$, is an isomorphism for $j \leqslant \operatorname{dim} A-$ $\operatorname{cod} B-k$, and a surjection for $j=\operatorname{dim} A-\operatorname{cod} B-k+1$ for any $a \in$ $A \cap B$ and its image $a^{\prime}$ in $\tilde{B}$. This is the basic technical result of the paper.

We then add the condition that the map $G \times A \rightarrow X$ induced by the group action is a fibre bundle. Under this additional condition we conclude from the result of the last paragraph that for all $a \in A \cap B$,

$$
\pi_{j}(A, A \cap B, a) \longrightarrow \pi_{j}(X, B, a)
$$

and

$$
\pi_{j}(B, A \cap B, a) \longrightarrow \pi_{j}(X, A, a)
$$

are isomorphisms for $j \leqslant \operatorname{dim} A-\operatorname{cod} B-k$ and surjections for $j=\operatorname{dim} A$ $-\operatorname{cod} B-k+1$.

Let $f: W \rightarrow Y$ be a holomorphic map from a connected compact complex manifold $W$ to a homogeneous complex manifold $Y$. Let $Z$ be a closed complex submanifold of $Y$. In Section 2 we apply the above by taking $X=W \times Y, A=W \times Z$, and $B$ equal to the graph of $f$. In this case the normal bundle of $B$ in $X$ is isomorphic to $f^{*} T_{Y}$. The result we obtain applies to not necessarily compact homogeneous manifolds. Specializing this result to a rational homogeneous projective manifold $W$, we obtain the result described at the beginning of this paper.

In the last section we give some examples including an application to the Gauss mapping.

We would like to thank the Max Planck Institut für Mathematik for their support. The first author would like to also thank the National Science Foundation [MCS 8200 629] for their support.

\section{$\S 1$. General results}

In this section we recall definitions and results that we need. We also prove a variant of the main result of [6] that is useful for our application.

We need the notion of $k$-ampleness in the sense of [4] for holomorphic vector bundles. Since our bundles are always spanned by global sections this notion takes a particularly simple form. Let $E$ be a holomorphic vector bundle on a compact complex manifold that is spanned at all points by global holomorphic sections. $E$ is $k$-ample if for each subvariety $Z \subseteq X$ such that $\left.E\right|_{Z}$ has a trivial quotient bundle, it is true that $\operatorname{dim} Z \leqslant k$. 
Throughout the rest of this section it is assumed that

a) $\rho: G \times X \rightarrow X$ is a real analytic action of a connected Lie group $G$ on a connected not necessarily compact complex manifold $X$ where for any $g \in G, \rho(g, x):\{g\} \times X \rightarrow X$ is a biholomorphism. To conform to the notion of $[6 ; \S 3]$, we write $x g$ for $\rho(g, x)$.

b) $A$ and $B$ are connected complex submanifolds of $X$ which have a non-empty intersection.

c) $B$ is compact and that the normal bundle of $B$ is both spanned by global sections at all points and $k$ ample for some $k \leq \operatorname{dim} A-\operatorname{cod} B$.

(1.1) Theorem. Let $G, X, B$ and $A$ be as above. Then for all $g \in G$, $A g \cap B$ is non-empty. Let $\tilde{B}$ denote the family of intersections of $B$ with G-translations of $A$ :

$$
\tilde{B}=\{(g, a) \in G \times A \mid a g \in B\} .
$$

If $k<\operatorname{dim} A-\operatorname{cod} B$ then the number of connected components of $A g \cap B$ is independent of $g \in G$. Further the map:

$$
\pi_{j}(A, A \cap B, a) \longrightarrow \pi_{j}\left(G \times A, \tilde{B}, a^{\prime}\right)
$$

induced by the inclusion $A \rightarrow\left(\mathrm{id}_{G}, A\right)$, is an isomorphism for $j \leqslant \operatorname{dim} A-$ $\operatorname{cod} B-k$, and a surjection for $j=\operatorname{dim} A-\operatorname{cod} B-k+1$ for any $a \in A$ $\cap B$ and its image $a^{\prime}$ in $\tilde{B}$.

Proof. To simplify notation, basepoints are suppressed. Our notation is chosen compatibly with $[6 ; \S 3]$. We let $\tilde{p}: \tilde{B} \rightarrow G$ denote the map induced by the product projection $p: G \times A \rightarrow G$.

Since $B$ is compact and the normal bundle of $B$ is spanned at all points by global sections and $k$ ample it follows from the main theorems of $[5, \S 7]$ that $X-B$ is $\operatorname{cod} B+k$ convex in the sense of AndreottiGrauert. We now use the main results of [6]. Our notation has been set up to agree with that of [6; Lemma (3.1.3), pg. 123]. The argument of that lemma applies here, except that instead of assuming that $G$ acts transitively, we assumed explicitly that $A \cap B$ is non-empty. From this argument we draw the conclusions that $\tilde{p}(\tilde{B})=G$ if $\operatorname{dim} A \geqslant \operatorname{cod} B+k$ and that $\tilde{p}$ is a $\operatorname{dim} A-\operatorname{cod} B-k$ quasi-fibration if $\operatorname{dim} A \geqslant \operatorname{cod} B+$ $k+1$. Note that $\tilde{p}(\tilde{B})=G$ implies that $A g \cap B$ is non-empty for each $g \in G$ and that the definition $[6 ;(2.1)]$ of a $\operatorname{dim} A-\operatorname{cod} B-k$ quasifibration implies that the number of connected components of $A g \cap B$ is independent of $g \in G$. 
From [6; Proposition (2.3)], we conclude that under the inclusion of $A \cap B$ in $\tilde{B}$ given by $A \rightarrow\left(\mathrm{id}_{G}, A\right)$ :

$$
\left\{\begin{array}{l}
\tilde{p}_{*}: \pi_{j}(\tilde{B}, A \cap B) \longrightarrow \pi_{j}(G) \text { is an isomorphism } \\
\text { for } j \leqslant \operatorname{dim} A-\operatorname{cod} B-k \text { and a surjection } \\
\text { for } j=\operatorname{dim} A-\operatorname{cod} B-k+1 .
\end{array}\right.
$$

Associated to the commutative square:

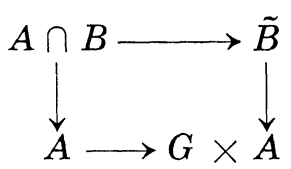

we have two exact sequences of homotopy groups:

$$
\begin{aligned}
& \pi_{j}(\tilde{B}, A \cap B) \longrightarrow \pi_{j}(G \times A, A \cap B) \longrightarrow \pi_{j}(G \times A, \tilde{B}) \longrightarrow \pi_{j-1}(\tilde{B}, A \cap B) \\
& \pi_{j}(A, A \cap B) \longrightarrow \pi_{j}(G \times A, A \cap B) \longrightarrow \pi_{j}(G \times A, A) \longrightarrow \pi_{j-1}(A, A \cap B)
\end{aligned}
$$

From $(*)$ above we conclude that the composition:

$$
\pi_{j}(\tilde{B}, A \cap B) \longrightarrow \pi_{j}(G \times A, A) \approx \pi_{j}(G)
$$

of

$$
\pi_{j}(\tilde{B}, A \cap B) \longrightarrow \pi_{j}(G \times A, A \cap B)
$$

and

$$
\pi_{j}(G \times A, A \cap B) \longrightarrow \pi_{j}(G \times A, A)
$$

is an isomorphism for $j \leqslant \operatorname{dim} A-\operatorname{cod} B-k$ and a surjection for $j=$ $\operatorname{dim} A-\operatorname{cod} B-k+1$.

A standard diagram chase on the above exact sequences combined with the $\left({ }^{*}\right)$ implies that the composition

$$
\pi_{j}(A, A \cap B) \longrightarrow \pi_{j}(G \times A, \tilde{B})
$$

of

$$
\pi_{j}(A, A \cap B) \longrightarrow \pi_{j}(G \times A, A \cap B)
$$

and

$$
\pi_{j}(G \times A, A \cap B) \longrightarrow \pi_{j}(G \times A, \tilde{B})
$$

is an isomorphism for $j \leqslant \operatorname{dim} A-\operatorname{cod} B-k$ and a surjection for $j=$ $\operatorname{dim} A-\operatorname{cod} B-k+1$. This finished the proof of the theorem. 
(1.1.1) Remark. Proposition (1.1) of [6] applied to our situation shows that if $\operatorname{dim} A \geqslant \operatorname{cod} B+k$, then the map $\tilde{B} \rightarrow G$ is either empty or onto, i.e. if $A g \cap B$ is non-empty for one $g \in G$ then it is non-empty for all $g \in G$.

To proceed further we need some extra control over the group action. Let $\rho_{A}: G \times A \rightarrow X$ denote the restriction of to $G \times A$.

(1.2) TheOREM. In addition to the hypotheses of Theorem (1.1) assume that the map $\rho_{A}: G \times A \rightarrow X$ given by the group action is surjective and $a$ fibre bundle. Then for any $a \in A \cap B$ :

$$
\pi_{j}(A, A \cap B, a) \longrightarrow \pi_{j}(X, B, a)
$$

and

$$
\pi_{j}(B, A \cap B, a) \longrightarrow \pi_{j}(X, A, a)
$$

are isomorphisms for $j \leqslant \operatorname{dim} A-\operatorname{cod} B-k$ and surjections for $j=\operatorname{dim} A$ $-\operatorname{cod} B-k+1$.

Proof. Note that $\tilde{B}=\rho_{A}^{-1}(B)$. Thus $\tilde{B} \rightarrow B$ is a pullback of the fibre bundle $\rho_{A}$ under the inclusion of $B$ into $X$. From this we conclude by a standard argument that the map

$$
\pi_{j}(G \times A, \tilde{B}) \longrightarrow \pi_{j}(X, B)
$$

induced by $\rho_{A}$ is an isomorphism for all $j \geqslant 0$. Combined with the conclusion of the last theorem we have that:

$$
\pi_{j}(A, A \cap B) \longrightarrow \pi_{j}(X, B)
$$

is an isomorphism for $j \leqslant \operatorname{dim} A-\operatorname{cod} B-k$ and a surjection for $j=$ $\operatorname{dim} A-\operatorname{cod} B-k+1$.

This is half of the theorem. To get the other half, write down the homotopy exact sequences associated to the commutative diagram:

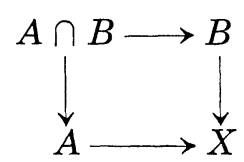

Using (\#) the argument proceeds exactly as in Theorem (1.1).

\section{§2. The main theorem}

(2.1) TheOREM. Let $f: W \rightarrow Y$ be a holomorphic map from a connected compact complex manifold $W$ to a connected homogeneous not necessarily 
compact complex manifold $Y$. Assume that $Y$ is of the form $G / V$ where $G$ is a simply connected group of biholomorphisms of $Y$ and $V$ is a connected subgroup of $G$. Assume that $f^{*} T_{Y}$, the pullback to $W$ of the holomorphic tangent bundle of $Y$, is $k$ ample (in the sense of [4]; see $\S 1$ ). Let $Z$ be a connected closed complex submanifold of $Y . \quad$ Let $d=\operatorname{dim} W-\operatorname{cod} B-k$. If $d>0$ then $f^{-1}(Z)$ is connected and for all $a \in f^{-1}(Z)$

$$
f_{*}: \pi_{j}\left(W, f^{-1}(Z), a\right) \longrightarrow \pi_{j}(Y, Z, f(a))
$$

is an isomorphism if $j \leqslant d$, and a surjection if $j=d+1$.

Proof. In the following proof we suppress basepoints for simplicity of notation.

Let $X$ and $A$ denote the manifolds $W \times Y$ and $W \times Z$ respectively. Let $B$ denote the graph of $f$ in $X$. Note that the normal bundle of $B$ in $X$ is isomorphic to $f^{*}\left(T_{Y}\right)$ where $T_{Y}$ is the holomorphic tangent bundle of $Y$. Since $Y$ is homogeneous it follows that $T_{Y}$ and hence $f^{*}\left(T_{Y}\right)$ is spanned by global holomorphic sections. Therefore the normal bundle of $B$ in $X$ is $k$ ample for some $k$. From the homogeneity of $Y$ and the definition of $A$ and $B$ it follows that $A g \cap B$ is non-empty for some $g \in G$.

Note that map $\rho_{A}: G \times A \rightarrow X$ given by the group action $\rho$ is a fibre bundle. Note further that the fibre, $F$, of this map is a fibre bundle over $Z$ with isotropy group $V$ as fibre.

Since $G \times A \rightarrow X$ is a fibre bundle, we conclude for Theorem (1.2) that

$$
\pi_{j}(B, A \cap B) \longrightarrow \pi_{j}(X, A)
$$

is an isomorphism for $j \leqslant \operatorname{dim} A-\operatorname{cod} B-k$ and surjection for $j=\operatorname{dim} A$ $-\operatorname{cod} B-k+1$. Note that $\operatorname{dim} A-\operatorname{cod} B-k=\operatorname{dim} W+\operatorname{dim} Z-$ $\operatorname{dim} Y-k$. Since

$$
\pi_{j}(B, A \cap B)=\pi_{j}\left(W, f^{-1}(Z)\right), \quad \pi_{j}(X, A)=\pi_{j}(Y, Z),
$$

and the homomorphism $\left({ }^{*}\right)$ corresponds to

$$
f_{*}: \pi_{j}\left(W, f^{-1}(Z)\right) \longrightarrow \pi_{j}(Y, Z)
$$

we conclude that $f_{*}$ is an isomorphism for $j \leqslant d$, and a surjection for $j=d+1$.

All that remains is to show that $f^{-1}(Z)$ is connected. Since $\rho_{A}$ is a fibre bundle, so also is the map $\tilde{B}=\rho_{A}^{-1}(B) \rightarrow B$ given by the restriction 
of $\rho_{A}$ to $\tilde{B}$. Since the both fibre $F$ of $\rho_{A}$ and $B$ are connected, it follows that $\tilde{B}$ is connected. Assuming that $\operatorname{dim} Z>d$ it follows from Theorem (1.1) that $\tilde{B} \rightarrow G$ factors as $\tilde{B} \rightarrow M$ and $M \rightarrow G$ where $\tilde{B} \rightarrow M$ has connected fibres and $M \rightarrow G$ is a covering. Since $G$ is simply connected and $A \cap B$ is the fibre if $\tilde{B} \rightarrow G$ over $\mathrm{id}_{G}$, we conclude that $A \cap B$ is connected.

(2.1.1) Remark. It follows from Remark (1.1.1) that $f^{-1}(Z)$ is nonempty if $d \geqslant 0$.

The following proposition is an immediate corollary of the above theorem. We designate it a theorem because it is the main result of this paper.

(2.2) Theorem. Let $f: W \rightarrow Y$ be a holomorphic map from a connected compact complex manifold $W$ to be a connected rational homogeneous projective manifold $Y$. Assume that $f^{*} T_{Y}$, the pullback of the holomorphic tangent bundle of $Y$, is $k$ ample. Let $Z$ be a connected complex submanifold of $Y$. Let $d=\operatorname{dim} W-\operatorname{cod} Z-k$. If $d>0$ then $f^{-1}(Z)$ is connected and for all $a \in f^{-1}(Z)$

$$
f_{*}: \pi_{j}\left(W, f^{-1}(Z), a\right) \longrightarrow \pi_{j}(Y, Z, f(a))
$$

is an isomorphism if $j \leqslant d$, and a surjection if $j=d+1$.

(2.1.2) Remark. If the holomorphic tangent bundle, $T_{Y}$, is $t$ ample, and if $m=\max \left\{\operatorname{dim} f^{-1}(y) \mid y \in Y\right)$, then $k \leqslant t+m$. This is an immediate consequence of the definition of $k$ ampleness.

\section{§ 3. Examples}

In this section we show how to use the results of this paper. Throughout this section we suppress basepoints.

The following is a restatement of Theorem (2.2) that follows from an elementary diagram chase.

(3.1) Theorem. Let $f, W, Y, Z$, and $d$ be as in Theorem (2.2). Assume that $d>0$. Let $i$ denote the inclusion of $f^{-1}(Z)$ in $W$ and let $j$ denote the inclusion of $Z$ in $Y$. Then there is an exact sequence:

$$
\pi_{d}\left(f^{-1}(Z)\right) \stackrel{a}{\longrightarrow} \pi_{d}(W) \oplus \pi_{d}(Z) \stackrel{b}{\longrightarrow} \pi_{d}(Y) \longrightarrow \pi_{d-1}\left(f^{-1}(Z)\right) \longrightarrow \cdots
$$

Here $a=i_{*}+f_{*}$ and $b=f_{*}-j_{*}$. 
The above is very useful for constructing examples of projective varieties with unusual homotopy groups. To illustrate this we restrict for simplicity to the previously known case of the theorem [2] when $Y=P^{4}$ and $Z$ is a smooth surface. We assume that $\operatorname{dim} W=4$ and $f$ is a finite to one surjection. The above exact sequence becomes:

$$
\pi_{2}\left(f^{-1}(Z)\right) \rightarrow \pi_{2}(W) \oplus \pi_{2}(Z) \rightarrow \pi_{2}\left(P^{4}\right) \rightarrow \pi_{1}\left(f^{-1}(Z)\right) \rightarrow \pi_{1}(W) \oplus \pi_{1}(Z) \rightarrow 0 .
$$

(3.1.1) EXAMPLE. Let $W$ be an arbitrary 4 dimensional Abelian variety. There exists a smooth surface $S \subseteq W$ with the properties:

a) the canonical bundle of $S$ is ample and $c_{1}^{2}(S) / c_{2}(S)=5 / 3$,

b) there is an exact sequence

$$
0 \longrightarrow Z \longrightarrow \pi_{1}(S) \longrightarrow Z^{12} \longrightarrow 0
$$

where $Z^{12}$ denotes the direct sum of 12 copies of the integers, $Z$.

To construct this example let $f: W \rightarrow P^{4}$ be any finite to one surjection. Let $Z \in P^{4}$ be a general translate under the projective linear group of the famous Horrocks-Mumford Abelian surface of degree 10. The assertion b) is immediate from (3.1) above. The assertion of a) is a direct calculation.

There are many other interesting manifolds to pullback, e.g. $\boldsymbol{P}^{2}$ embedded into $\boldsymbol{P}^{5}$ by the Veroness embedding.

In Theorems (2.1) and (2.2) we use the ampleness of $f^{*} T_{Y}$ instead of simply using the sum of the ampleness of $T_{Y}$ plus the maximum fibre dimension of $f$. To show that this is a true improvement we conclude with a new type of Lefschetz theorem. Let $\operatorname{Gr}(n, r)$ denote the Grassmannian of quotient $\boldsymbol{C}^{r}$ 's of $\boldsymbol{C}^{n}$.

(3.2) Theorem. Let $E$ be a holomorphic vector bundle on a compact complex manifold, $W$. Assume that $E$ is spanned at all points by an dimensional vector space $V$ of global section. Let $F$ be the kernel of the surjective bundle map

$$
W \times V \longrightarrow E \longrightarrow 0
$$

given by evaluation. Let $f: W \rightarrow \operatorname{Gr}(n, \operatorname{rk} E)$ be the map associated to (\#). Let $Z$ be any compact connected complex submanifold of $\operatorname{Gr}(n, \mathrm{rk} E)$ and assume that $E \otimes F^{*}$ is $k$ ample. Then

$$
\pi_{j}\left(W, f^{-1}(Z)\right) \longrightarrow \pi_{j}(\operatorname{Gr}(n, \operatorname{rk} E), Z)
$$


is an isomorphism for $j \leqslant \operatorname{dim} W-\operatorname{cod} Z-k$ and a surjection for $j=\operatorname{dim} W$ $-\operatorname{cod} Z-k+1$.

Proof. Let $Y=\operatorname{Gr}(n, \operatorname{rk} E)$ and note that $f^{*}\left(T_{Y}\right) \approx F^{*} \otimes E$. The theorem now follows from Theorem (2.2).

(3.2.1) Remark. Let $E$ be a $k$ ample vector bundle on a compact complex manifold $W$. Assume that $E$ is spanned by global sections and that $B$ is the zero set of a holomorphic section of $E$. The standard Lefschetz Theorem for a $k$ ample vector bundle spanned at all points by global sections (which follows for example from the main theorem of [7]) asserts that

$$
\pi_{j}(W, B)=0 \quad \text { for all } j \leqslant \operatorname{dim} W-\operatorname{rk} E-k .
$$

This follows also from the above result. To see this let $E, W$ and $B$ be as in this remark and let $V, F$, and $f$ be as in the above theorem. For an appropriate codimension one subspace of $V, B=f^{-1}(\operatorname{Gr}(n-1, \operatorname{rk} E))$. Note that $\pi_{j}(\operatorname{Gr}(n, \operatorname{rk} E), \operatorname{Gr}(n-1, \operatorname{rk} E))=0$ for $j \leqslant 2(n-\operatorname{rk} E)-1$. Noting that $n \geqslant \operatorname{dim} W+\operatorname{rk} E-k$ we see that $\pi_{j}(\operatorname{Gr}(n, \operatorname{rk} E), \operatorname{Gr}(n-1$, $\operatorname{rk} E))=0$ for $j \leqslant \operatorname{dim} W-\operatorname{rk} E-k$. Combining this with the above theorem gives $(*)$.

The above has an interesting application to the Gauss mapping. Let $W$ be an $r$ codimensional projective submanifold of $\boldsymbol{P}^{n-1}$ not contained in any linear $\boldsymbol{P}^{n-2}$. Then the Gauss mapping $f: W \rightarrow \operatorname{Gr}(n, r)$ is the map associated to the evaluation mapping

$$
W \times V \longrightarrow E \longrightarrow 0
$$

where

$$
V=\left.\Gamma\left(\boldsymbol{P}^{n-1}, \mathcal{O}_{\boldsymbol{P}^{n-1}}(1)\right)^{*}\right|_{W}
$$

and $E=N_{W}(-1)$, the normal bundle of $W$ in $\boldsymbol{P}^{n-1}$ twisted by $\mathcal{O}_{\boldsymbol{P}^{n-1}}(-1)$. The kernel of $(\#)$ is $J_{1}\left(W, \mathcal{O}_{W}(1)\right)^{*}$, the dual of the first jet bundle of the restriction to $W$ of the hyperplane section bundle $\mathcal{O}_{p^{n-1}}(1)$. Therefore for this map

$$
f^{*} T_{\mathrm{Gr}(n, r)} \approx J_{1}\left(W, \mathcal{O}_{W}(1)\right) \otimes N_{W}(-1)
$$

which is $k$ ample if either $J_{1}\left(W, \mathcal{O}_{W}(1)\right)$ or $N_{W}(-1)$ is $k$ ample. We thus get a first result towards answering the question posed in $[2 ; 10.5]$. 
(3.3) Theorem. Let $f: W \rightarrow \operatorname{Gr}(n, r)$ be the Gauss mapping associated to an $r$ codimensional projective submanifold of $\boldsymbol{P}^{n-1}$. Assume that $J_{1}\left(W, \mathcal{O}_{W}(1)\right)$ or $N_{W}(-1)$ or more generally $J_{1}\left(W, \mathcal{O}_{W}(1)\right) \otimes N_{W}(-1)$ is $k$ ample. Let $Z$ be a connected complex submanifold of $\operatorname{Gr}(n, r)$. If $\operatorname{dim} W \geqslant \operatorname{cod} Z$ $k, f^{-1}(Z)$ is non-empty. If $\operatorname{dim} W>\operatorname{cod} Z+k$, then $f^{-1}(Z)$ is connected and

$$
f_{*}: \pi_{j}\left(W, f^{-1}(Z)\right) \longrightarrow \pi_{j}(\operatorname{Gr}(n, r), Z)
$$

is an isomorphism for $j \leqslant \operatorname{dim} W-\operatorname{cod} Z-k$ and a surjection for $j=$ $\operatorname{dim} W-\operatorname{cod} Z-k+1$.

It is easy to check that $J_{1}(W, L)$ is ample if $L$ is the square of a very ample line bundle. It is not hard to check that unless $W$ is projective space and $L=O(1)$, it follows that $J_{1}(W, L)$ is $\operatorname{dim} W-1$ ample.

The theorem analogous to (3.3) holds for the Gauss mapping associated to a codimension $r$ submanifold, $W$, of an $n$ dimensional Abelian variety, $A$. Here the $k$ ampleness hypothesis is changed to

Assume that $T_{W}^{*}$, or $N_{W}$, or more generally $T_{W}^{*} \otimes N_{W}$ is $k$ ample, where $N_{W}$ is the normal bundle of $W$ in $A$.

Since there is an easy criterion [4] for the $k$ ampleness of $N_{W}$ based on a result of Hartshorne, this result is easily applied.

\section{REFERENCES}

[1] G. Faltings, Formale Geometrie und homogene Räume, Invent. Math., 64 (1981), 123-165.

[2] W. Fulton and R. Lazarsfeld, Connectivity and its applications in algebraic geometry, Lecture Notes in Mathematics, 862 (1981), 26-92 Springer-Verlag, New York.

[ 3 ] N. Goldstein, Ampleness and connectedness in complex $G / P$, Trans. Amer. Math. Soc., 274 (1982), 361-373.

[4] A. J. Sommese, Submanifolds of Abelian varieties, Math. Ann., 233 (1978), 229256.

[ 5 ] - Concavity theorems, Math. Ann., 235 (1978), 37-53.

[6] - Complex subspaces of homogeneous complex manifolds II-Homotopy results, Nagoya Math. J., 86 (1982), 101-129.

[ 7] —, A convexity theorem, Proceedings of Symposia in Pure Math., 40 (1983), Part 2, 497-505.
A. J. Sommese
Department of Mathematics
University of Notre Dame 
Notre Dame, Indiana 46556

U.S.A.

A. Van de Ven

Department of Mathematics

University of Leiden

Leiden, The Netherlands 